\title{
Visible light communication-based traffic control of autonomous vehicles at multi-lane roundabouts
}

\author{
Maged Fakirah', Supeng Leng ${ }^{1 *} \mathbb{D}$, Xiaosha Chen ${ }^{1}$ and Jihua Zhou ${ }^{2}$
}

\begin{abstract}
Roundabouts are a form of circular intersections adopted to secure road safety of the vehicles. However, collisions are not prevented entirely even when using roundabouts, since accidents may happen at specific intersection points leading to either traffic congestions or serious accidents. In this paper, a visible light communication (VLC)-based collision avoidance scheme is proposed to coordinate autonomous vehicles in traversing roundabouts, which has high effectiveness in vehicular environments. In particular, roadside units (RSUs) are deployed at the roundabout entrances to coordinate the vehicles in a vehicle-to-infrastructure (V2I) mode. By adopting the synchronization approach, vehicles can pass the roundabout simultaneously if their paths are concurrent with each other. Otherwise, vehicles are prioritized according to their arrival time and reasonable decelerations are applied to waive potential conflicts. Simulation results showed that our proposed approach satisfies the roundabout traffic demands in terms of concurrency, safety, and time utilization as vehicles are strongly recommended to decelerate in only $22 \%$ of the cases studied in different scenarios.
\end{abstract}

Keywords: Autonomous vehicles, Collision avoidance, ITS, Roundabout, Safety, Synchronization, VLC

\section{Introduction}

Road safety has been one of the vital topics studied in Intelligent Transportation Systems (ITS) as serious accidents cause loss of lives and destruction of infrastructures [1]. Nevertheless, prominent safety features have become possible due to advanced information and communications technologies [2, 3]. Accordingly, autonomous vehicles are an effective solution that could increase both the driving safety and ride comfort besides enhancing the road traffic flow [4].

Road intersections are a fertile place for accidents since conflicts occur when two vehicles (or more) approach the same point simultaneously $[5,6]$. To improve the traffic safety on road intersections, roundabouts have been widely adopted within the traffic infrastructure, for which

\footnotetext{
*Correspondence: spleng@uestc.edu.cn

${ }^{1}$ School of Information \& Communication Engineering, University of Electronic Science and Technology of China (UESTC) Chengdu, China
}

Full list of author information is available at the end of the article they increase the traffic throughput and reduce the time delay, in addition to the limited amount of maintenance through a long latency. Roundabouts are sort of circular intersections, where vehicles enter the circle when a gap exists in the circulating area. After that, they pass the lanes in a clockwise direction (in countries with right-hand traffic). Vehicles must comply with the offside-priority rule so that circulating vehicles have priority over those entering the traffic stream [7]. To secure more efficiency, multilane roundabouts are applied in heavy traffic intersections with high-speed approaches [8].

Nevertheless, multi-lane roundabouts do not prevent accidents entirely as serious collisions may happen due to several factors, such as driving in high velocities with inadequate deceleration, the unpredictability of the other vehicles' destinations, and finally, noncompliance with the priority-to-circle rules. Figure 1 shows some potential conflicts more likely to happen in the daily traffic among both autonomous and human-driven vehicles: (1) The 


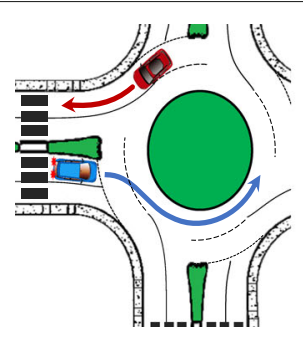

(a) Entering vehicle stops unnecessarily.

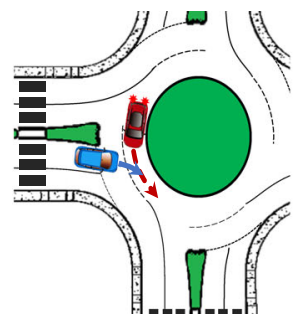

(b) Circulating vehicle advancesed by the entering vehicle.

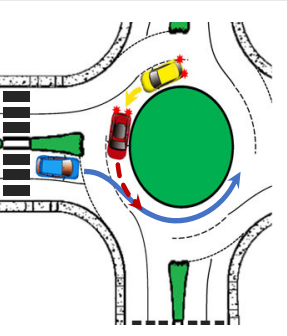

(c) Priority reversal given to the waiting vehicle.

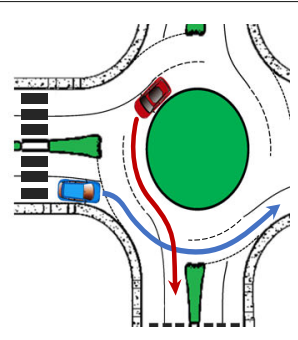

(d) Entering vehicle clashes with the exiting vehicle.

Fig. 1 Roundabout operations

entering vehicle stops while there is an existed gap sufficient for the entrance. (2) The entering vehicle advances the circulating vehicle, even if the gap is insufficient, forcing it to stop moving. (3) The circulating vehicle gives its priority on the road to the vehicle waiting to enter the roundabout (referred to as priority reversal). (4) The circulating vehicle heads out beside a vehicle entering the roundabout causing confusion for both drivers.

This issue becomes more serious when involving autonomous vehicles in the traffic since although they can regulate their positions automatically, it is difficult to make intelligent decisions in complicated and dense driving environments such as multi-lane roundabouts. Furthermore, it is impossible to anticipate other road user's behavior or even to transfer the control to a human driver in case of risky events, which led to the need for an auxiliary system to coordinate the autonomous vehicles and contribute to their safety. In this case, the vehicle safely traverses the roundabout with the assistance of the auxiliary system considering the traffic situation and the applicable road priority. If it reaches the system limit, it keeps driving at a reasonable speed until it leaves the roundabout successfully $[9,10]$.

For the vehicle-to-infrastructure (V2I) communication, signal interference and long transmission delays are potential through the data exchange, which can affect the comprehensive system performance. Thus, using conventional radio frequency (RF) waves is not ideal for the communication with autonomous vehicles as they demand a reliable and super-high network connectivity to meet the safety requirements $[11,12]$. Furthermore, due to its distinguishing merits such as the less complexity, low energy, and cost-effectiveness [13], the visible light communication (VLC) is utilized as an alternative solution to the $\mathrm{RF}$ communications to receive and send the vehicle and traffic information from and to the correlated RSU.

Accordingly, a VLC-based assistive approach is proposed to coordinate autonomous vehicles while passing the roundabout, by supplying them with the information of any instant traffic changes, which reserve adequate time to take appropriate reactions and precise decisions. However, three challenges need to be faced for the design of our collision avoidance system: (1) Safety against the time delay. It is challenging to maintain the balance between required safety and the time delay for communication and decision, since long time delays may lead to driver irritation, stress, and frustration that may generate more offensive driving behaviors besides slowing traffic flow. (2) The roundabout operational conditions. Some factors have to be taken into consideration when using the VLC in the practical driving environment, such as the transmitters' locations and the effective transmission distance [14]. Thus, it is challenging to consider these factors accurately when determining broadcast area and the system workspace. (3) The ambient light. In outdoor environments, the VLC technique exposes to some sources of external noise, i.e., ambient road lights, the sunlight, and finally, the noise associated with light carrying the warning signal itself. These noises may affect the system performance in terms of the signal-to-noise ratio (SNR) [15].

The main contributions of our system are briefly highlighted as follows:

- The focus on the multi-lane roundabouts and mapping the traffic problem into a typical synchronization approach. Hence, a centralized algorithm based on the VLC technique is applied in the V2I mode.

- The signal processing complexity at the receiver is significantly reduced by adopting the VLC technique with the aid of the intensity modulation with direct detection (IM/DD). In addition, the SNR value is investigated in each change of vehicles' position to cope with the challenge of ambient noise and to assess the performance of the system accurately.

- Extensive simulations based on various mobility scenarios are conducted. The simulation results confirmed that the system proposed can assist the autonomous vehicles in taking appropriate reactions according to different driving conditions. 
The remaining parts of this paper are organized as follows: Section 2 briefly presents some research about traffic management. Section 3 focuses on the analysis of the system proposed. The numerical calculations required to estimate the system performance are illustrated in Section 4 along with the analysis and discussion of the results. Finally, Section 5 is reserved for both the conclusion and future work.

\section{Related work}

To deal with these issues, some research related have been carried out following various approaches rather than adopting the standard traffic light system. In [16], a decentralized vehicle-to-vehicle (V2V) approach, which connects human-driven vehicles together, has been presented to eliminate the potential overlaps out of vehicular paths. Assuming that the vehicles head toward the roundabout in steady velocities, that is inapplicable. The approach presented solves from 60 to $80 \%$ of possible conflicts with $20-40 \%$ of packet loss ratio. In [17], the authors presented a new protocol for collision detection in autonomous vehicles based on $\mathrm{V} 2 \mathrm{~V}$ communications. The protocol proposed has reduced the delay and influenced, positively, to avoid collisions. However, it has been only tested on autonomous vehicles, not on human-driven vehicles. The authors in [18] designed a control model for multi-line roundabouts by adopting both cameras and Wireless Sensor Networks (WSN). This model aims to ensure that the current number of vehicles in the roundabout is below the vehicles' optimal number. Results exhibited that the delay is reduced, effectively, during the peak hour.

Additionally, different process management approaches in operating systems design have been utilized for vehicular coordination at intersections along with conventional transmission means [5, 19, 20]. Literature [19] designed two novel algorithms, i.e., a centralized and distributed algorithm, to control the traffic at intersections. They modelled the competition problem at the intersection as a Vehicle Mutual Exclusion for Intersections Problem (VMEI) and compared the algorithms proposed with the conventional traffic light system to show its outperformance. However, they confirmed that deploying the centralized algorithm is not feasible at all times due to the high cost of additional devices required.

Furthermore, various recent studies discussed the traffic and resource allocation issues and presented some algorithms to improve the existing once. In [21], the main focus was on the edge offloading associated with fog computing. The authors studied the tradeoff between the service latency and power consumption of the industrial traffic and designed a new model to depict the desire of traffic mobile users to share their resources with other users. After that, two reinforcement learning scheduling algorithms have been proposed to address the formulated offloading cost-minimization problem. The authors in [22] analyzed some factors such as the transmission rate and cache allocation in content-centric IoT and studied their impact on the QoE. Then, they proposed a deep reinforcement learning algorithm to solve the formulated resource allocation problems and enhance the QoE precision accordingly. Wang et al. [23] present an overview of the ongoing research in resource allocation and efficient scheduling. It elaborated on the state-of-the-art techniques of the cluster framework algorithms and classified them according to different principles. Finally, it illuminated the distributed systems design principles by analyzing the properties of five desirable categories.

On the other hand, adopting the VLC technique has become a vital solution adopted in many state-of-the art research to solve traffic issues. In the work [24], authors have performed a comparative analysis of a crowdsensing framework that utilizes the VLC technique only with that utilizing VLC and IEEE 802.11p, simultaneously, considering the packet delivery ratio. Results asserted the performance improvement when utilizing both techniques, as each one is complementary to each other, especially in dense traffic scenarios. Cen Liu [25] estimated the vehicular VLC network (V2LC) based on a developed platform to study its resiliency, capability, and feasibility against the noise and interference of the visible light. The results proved that V2LC can serve the vehicles' network applications as it overcomes the packet collisions' impact when reaching vehicles in the high traffic density utilizing the inter-vehicle gaps and multiple paths available. The authors in [26] developed V2LC, a system that combines both smartphone cameras and vehicle lighting. The system adopts the Undersampled Frequency Shift ON-OFF Keying modulation (UFSOOK) for transmission decoding. In the system proposed, smartphone cameras are considered as receivers and vehicles rear light LEDs are considered as transmitters. The results emphasized that the system is reliable, for which it can resist the interference and transmission noise in various tested scenarios. Nevertheless, these solutions have been dedicated to traffic issues with some deficiencies, as they do not satisfy all the requirements of feasibility, flexibility, and complexity.

\section{Methods}

\subsection{System model}

This section will present the basic assumptions, the channel models, and the evaluation factors in this paper. The quality of the system proposed is evaluated through the computation of both the SNR and bit error rate (BER) values. However, some related factors have to be identified first, i.e., the received power, channel DC gain, and the irradiation angle. 


\subsubsection{Received power}

The average received power on the receiver can be calculated with the DC gain as [27]:

$$
P_{r}=H(0) \times P_{t}
$$

where $P_{r}$ refers to the received power, while $H(0)$ refers to the channel DC gain, and finally, the transmitted power $P_{t}$ can be calculated using the following formula [27]:

$$
P_{t}=\lim _{T=\infty} \frac{1}{2 T} \int_{-T}^{T} X(t) d x
$$

where $X(t)$ refers to the periodic input power and $T$ refers to the time period.

To model the channel accurately, VLC channel is modelled with IM/DD as a linear additive white Gaussian noise (AWGN) channel, for which the modelling is given by [28]:

$$
y(t)=R x(t) \otimes h(t)+n(t)
$$

where $y(t), R, x(t), h(t)$, and $n(t)$ refer to the current received power, the photodiode responsivity, the instantaneous transmitted power, the response of the channel impulse, and the AWGN noise, respectively. Moreover, $\otimes$ means convolution.

\subsubsection{Channel DC gain}

Since the light propagates from the transmitter LED (considered as a Lambertian emitter) to the receiver through the transmission channel in a line of sight (LOS) path, the channel DC gain can be computed as [29]:

$$
H(\psi, \phi)= \begin{cases}\frac{(m+1) A \cos ^{m}(\phi) T s(\psi) g(\psi) \cos (\psi)}{2 \pi d^{2}}, & \leq \psi \leq \psi_{c} \\ 0, & \psi>\psi_{c}\end{cases}
$$

where $m$ denotes the order of the Lambertian emission, $A$ means the detector physical area, $\phi$ refers to the irradiation angle, $T s$ is the gain of optical filter, $\psi$ refers to the incidence angle at the transmitter, $g(\psi)$ is the gain of optical concentrator, and finally, $d$ means the space between the transmitter and the receiver.

In this paper, $\phi$ is being computed by the trigonometric relationship of the right-angled triangle as:

$$
\phi=\arccos \left(\frac{d_{\text {total }}}{d}\right)
$$

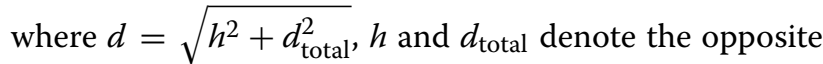
and adjacent and legs to the angle, respectively. See Fig. 2.

Moreover, to get the order of Lambertian emission, the following equation is used $[28,30]$ :

$$
m=\frac{-\ln (2)}{\ln \left(\cos \Phi_{1 / 2}\right)}
$$

where $\Phi_{1 / 2}$ denotes the transmitter semi-angle at half power.

Finally, the $g(\psi)$ can be computed by [28]:

$$
g(\psi)=\left\{\begin{array}{lc}
\frac{n^{2}}{\sin ^{2}\left(\psi_{c}\right)}, & 0 \leq \psi \leq \psi_{c} \\
0, & \psi>\psi_{c}
\end{array}\right.
$$

where $n$ denotes the air refractive index. On the other hand, $\psi_{c}$ denotes the receiver field of view (FOV).

\subsubsection{Quality of the system (SNR computation)}

Despite the factors discussed above significantly affect the design of the system, SNR plays a key role in measuring the wireless communications quality at the receiver. It defines the ratio of signal power to the background noise at a specific transmission point. Meaning that, the greater the signal strength, the larger the received data in the noise presence $[31,32]$. The SNR equation is expressed as:

$$
S N R=\frac{\left(R \cdot P_{r}\right)^{2}}{\sigma_{\text {total }}^{2}}
$$

where $\sigma_{\text {total }}^{2}$ refers to the total noise that consists of both shot noise and thermal noise, which are modelled as AWGNs [29]. Thus, $\sigma_{\text {total }}^{2}$ is given by [28]:

$$
\sigma_{\text {total }}^{2}=\sigma_{\text {shot }}^{2}+\sigma_{\text {thermal }}^{2}
$$

where $\sigma_{\text {shot }}^{2}$ and $\sigma_{\text {thermal }}^{2}$ denote the shot noise variance and the thermal noise variance, respectively. To get the shot noise, the following equation is used [28]:

$$
\sigma_{\text {shot }}^{2}=2 q B\left(R P_{r}+I_{B} I_{2}\right)
$$

where $q$ refers to the electronic charge, $B$ refers to the photodetector bandwidth, $I_{B}$ refers to the background current, and finally, $I_{2}$ refers to the noise bandwidth factor.

On the other hand, the thermal noise can be expressed as the combination of two parts, namely, the feedback resistor noise and the FET channel noise, respectively [33]. The $\sigma_{\text {thermal }}^{2}$ equation is given by [28]:

$$
\sigma_{\text {thermal }}^{2}=\frac{8 \pi K T_{k} \eta A I_{2} B^{2}}{G}+\frac{16 \pi^{2} K T_{k} \Gamma \eta^{2} A^{2} I_{3} B^{3}}{g_{m}}
$$

where $K$ refers to Boltzmann's constant, $T_{k}$ denotes the absolute temperature, $\eta$ means the photodetector fixed capacitance per unit area, $B$ is the photodetector bandwidth, $G$ denotes the voltage gain of open-loop, $\Gamma$ refers to FET channel noise factor, $I_{3}$ is the noise bandwidth factor, and finally, $g_{m}$ means the FET transconductance.

\subsubsection{Bit error rate (BER)}

The BER is the ratio between bit errors and the total number of bits transferred during a specific time period. It is commonly expressed in percentages without a particular measurement unit [32].

In this section, the BER performance for the On-Off Keying (OOK) modulation scheme is presented, as it is 


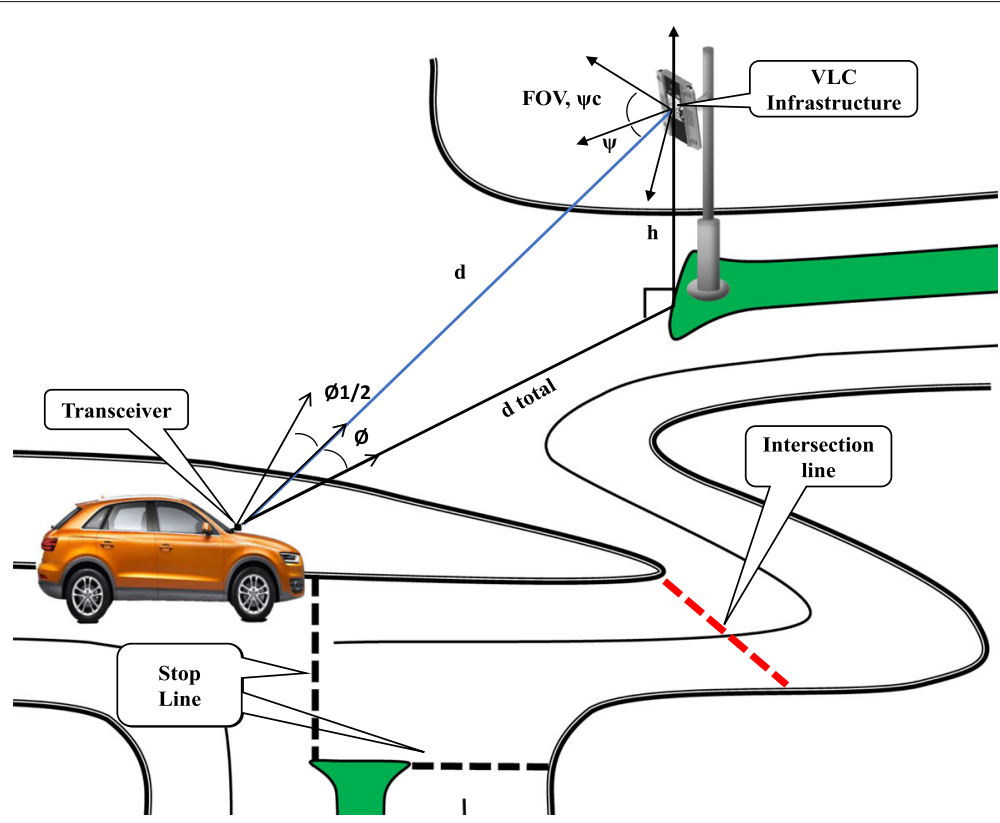

Fig. 2 Geometric model of propagation

the simplest modulation technique for IM/DD in Optical Wireless Communications (OWC) [34]. In OOK, 1 and 0 bits are encoded into pulses that arise through turning the LED on and off, respectively. Thus, a rectangular pulse with the intensity of $2 P$ (peak power) and a time period of $T=1 / R_{b}$ is emitted by the transmitter to indicate 1 bit, where $R_{b}$ refers to the bit rate. On the other hand, no pulse indicates a 0 bit $[28,35]$. Considering the AWGN, SNR can be formulated directly to BER by [31]:

$$
\mathrm{BER}_{\mathrm{OOK}}=Q \sqrt{\mathrm{SNR}}
$$

where $Q(x)=\frac{1}{2} \operatorname{erfc}\left(\frac{x}{\sqrt{2}}\right)$, for which erfc denotes the complementary error function.

\subsection{Problem formulation}

The roundabout traffic scenario will be discussed in this section. Furthermore, this section will define some key notations, which will be utilized in the following algorithm design.

As shown in Fig. 3, a typical multi-lane roundabout with four legs (north, west, south, and east), is considered. In each leg, there are two lanes with eight prospective paths leading to the intersection lines. For more simplicity, the paths are numbered from 1 to 8 and denoted by $P 1$ to $P 8$, respectively. These paths determine the vehicles passing on it, accordingly. Based on the ordinary roundabout traffic scenarios, to avoid potential collisions that arise due to bad driving behaviors (as mentioned early in this paper), the entering paths have to be locked while vehicles on the inner paths pass successfully. However, in this regard, some concurrent paths will be locked, simultaneously, affecting vehicles passing on these different paths, for which vehicles with non-conflicting paths should have the right to pass simultaneously.

As a result, an approach for traffic control of autonomous vehicles at multi-lane roundabouts, based on VLC centralized coordination is presented. In the approach proposed, vehicles' movements are tracked in real time to ensure more efficiency and safety of the vehicles being controlled by formulating the problem into a synchronization approach, where each vehicle moving towards the roundabout is considered as a process, which is being executed in the critical section. So how to control the traffic, efficiently, if there are many vehicles that aim to execute, at the same time, in the common resource, is the main concern of system proposed.

\subsubsection{Synchronization and potential conflicts}

The relationship between the eight paths can be obviously represented using the conflict graph as shown in Fig. 4. The vertices refer to the roundabout paths, and vehicles associated with them, while edges refer to the conflicts existed between paths. Hence, vehicles with edges connected are more likely to collide. As a consequence, the paths of conflicting vehicles conflict with each other as well. Contradictory, vehicles with indirect edges can pass the roundabout, simultaneously, where they are concurrent with each other. For instance, if a vehicle is passing through $P 4$, all conflicting paths have to be locked. Thus, approaching vehicles from $P 5$ and $P 6$ will be paused, consequently, and other vehicles on $P 1, P 2, P 3, P 7$, and $P 8$ will 


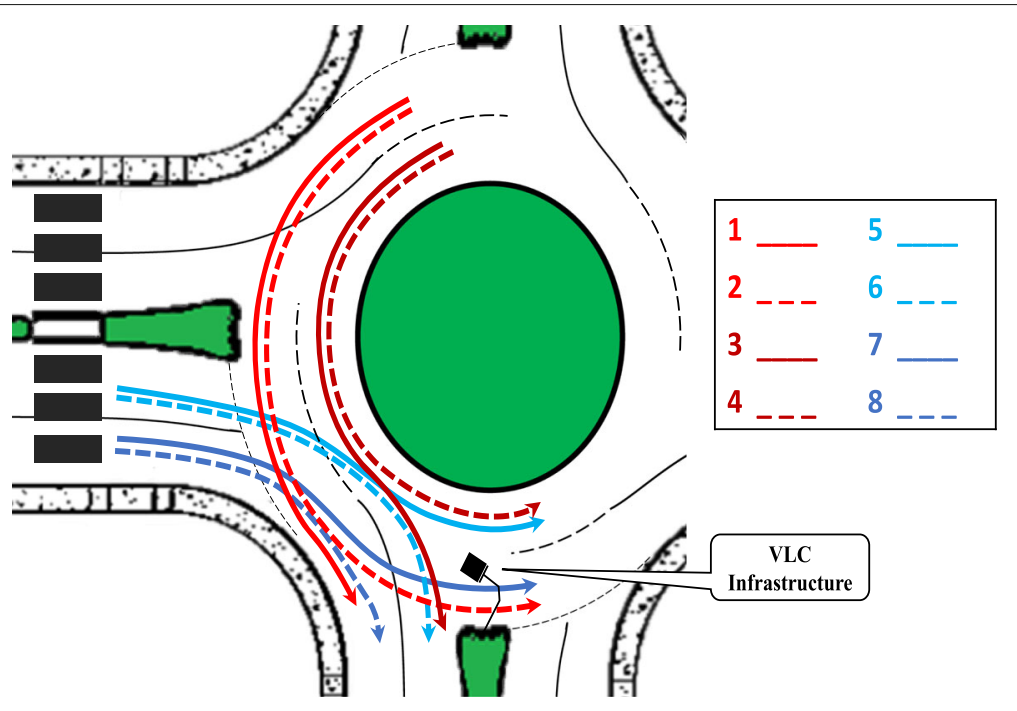

Fig. 3 Roundabout and lane conflicts

be able to pass, concurrently, because there will be no conflict between them and $P 4$. Table 1 explains the concurrent and conflicting relationships among paths.

\subsubsection{Communication network}

As Fig. 5 shows, the proposed system utilizes VLC technology as a communication medium between RSUs, circulating vehicles, and the entering vehicles. Assuming a multi-lane roundabout, two stopped lines are identified at $d_{\text {total }}$ meters of distance from $N$ RSUs installed at legs of the roundabout. Accordingly, the horizontal distance surrounded by the two stop lines and the peer RSU is identified as workspace area. On the other hand, the

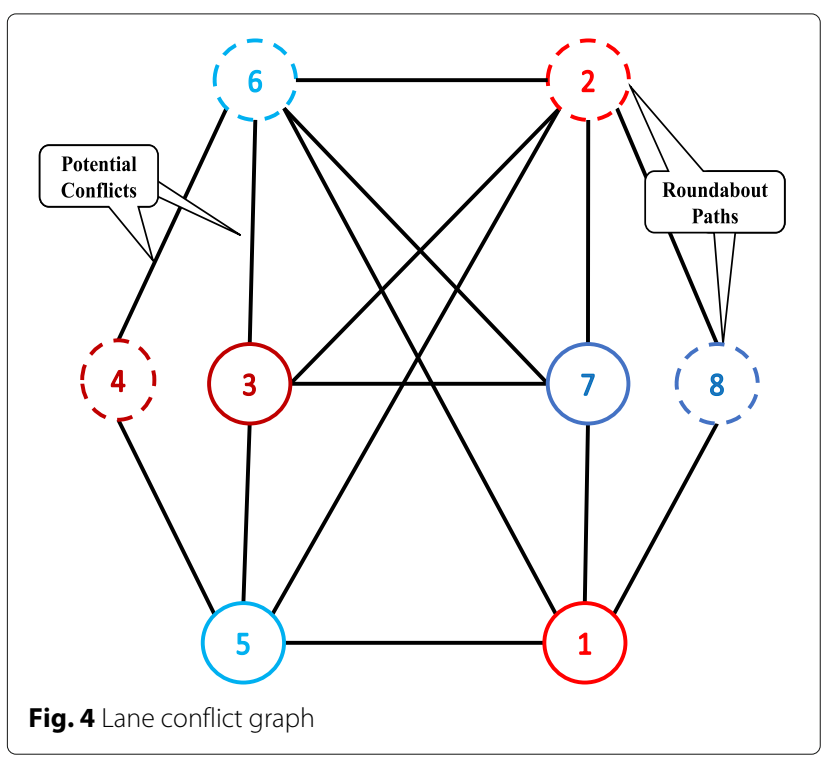

space beyond these stop lines is denoted as the broadcast area $d_{\mathrm{br}}$. This area allows the vehicles approaching the roundabout to decelerate properly within it.

\subsection{VLC-based collision avoidance algorithm}

The details and the analysis of the collision avoidance algorithm will be presented in this section. The communication interaction between the vehicle and RSU is illustrated in Fig. 6. In this regard, the vehicle that arrives at the roundabout broadcast area firstly $\left(V_{i}\right)$ sends a passing request to the system, containing the vehicle's traffic information (vehicle ID, arriving time, path number, and the direction) [36]. The Vehicle Identification Number (VIN), which serves as a unique identifier for each vehicle, is used to identify the vehicle ID. On the other hand, the Global Positioning System (GPS), which is the most used tool for determining positions in driving assisting systems, especially for autonomous vehicles [37], is used to determine the vehicle's location. Algorithm 1 summarizes clearly the procedure described above.

Table 1 Roundabout paths and locks correlated with them

\begin{tabular}{ll}
\hline Path of vehicle & Paths to be locked \\
\hline 1 & $5,6,7,8$ \\
2 & $3,5,6,7,8$ \\
3 & $2,5,6,7$ \\
4 & 5,6 \\
5 & $1,2,3,4$ \\
6 & $1,2,3,4,7$ \\
7 & $1,2,3,6$ \\
8 & 1,2 \\
\hline
\end{tabular}




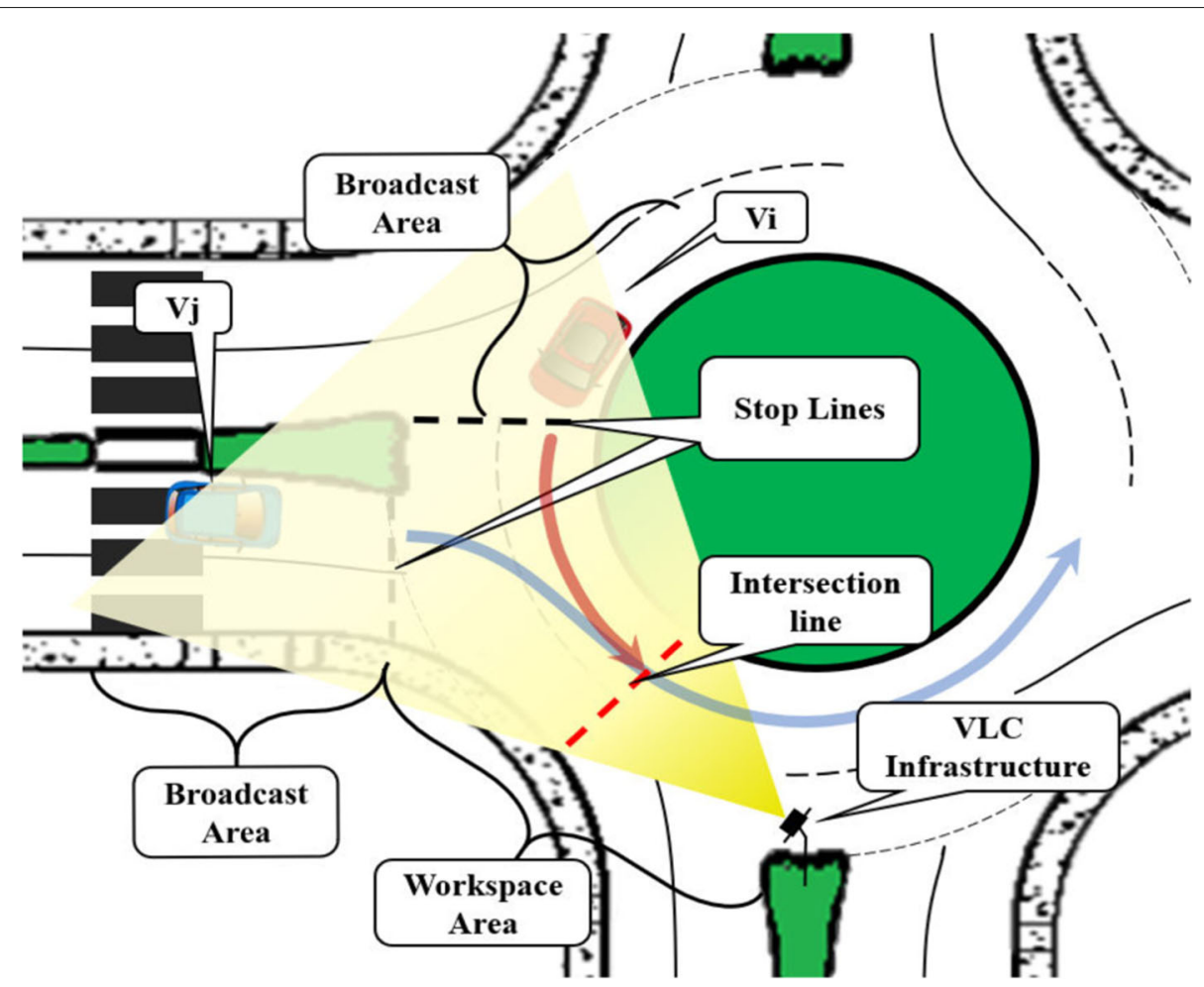

Fig. 5 Communication network and broadcast area

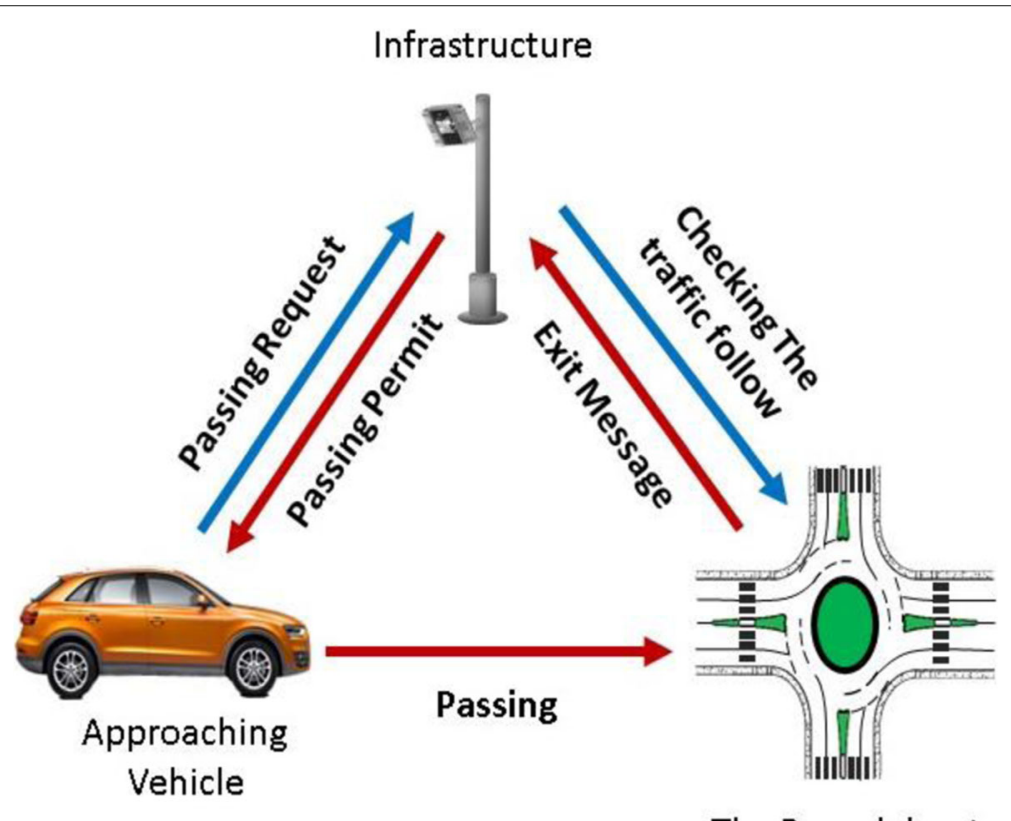

The Roundabout

Fig. 6 Interaction between the vehicle and infrastructure 


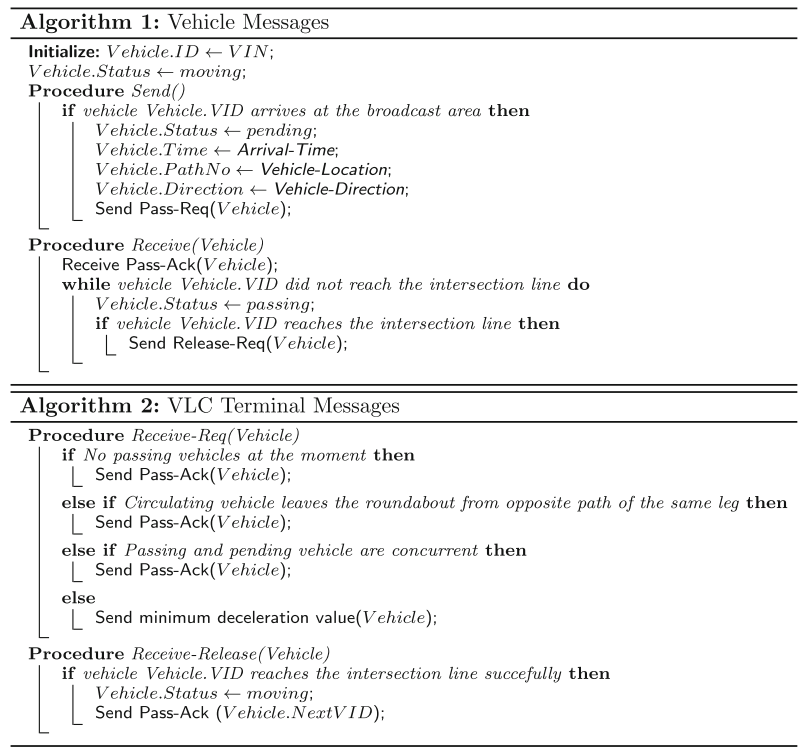

Based on the information received from the vehicle that arrived, the system will release an acknowledgment message, in the form of a warning signal and broadcast it within the broadcast area until $V_{i}$ arrives at the intersection point, successfully, and releases a confirmation message to the system, as explained in Algorithm 2.

To this end, if another vehicle $\left(V_{j}\right)$ reaches the broadcast area and sends a passing request, containing its traffic information, the system will check the conflict table to determine whether there is a potential conflict or not. If both paths are concurrent, $\left(V_{j}\right)$ will be granted passing permission. Otherwise, the system has to decide, based on the roundabout traffic flow, whether $\left(V_{j}\right)$ must pause, decelerate, or just keep progressing, as will be elaborated later in this paper. Finally, if both the circulating and entering vehicle reaches the broadcast area, at the same time, which might cause a deadlock, the circulating vehicle will be given the passing priority, according to the priorityto-circle rule. Figure 7 clarifies the whole processes of Algorithm 1 and Algorithm 2.

In our approach, the waiting time does not increase too much, in case of traffic density, as the vehicles are being served in a FIFO manner. Accordingly, vehicles are passing the roundabout as soon as they arrive based on the roundabout traffic situation.

Assuming that the first vehicle $V_{i}$ arrives at the roundabout stop line, the time $t_{i}$ demanded by $V_{i}$ to arrive at the intersection line is referred to by $t_{i}=\frac{d_{\text {total }}-d x}{u}$, where $d x$ denotes the area between the intersection line and VLC infrastructure and $u$ denotes the roundabout minimum circulating velocity, for both $V_{i}$ and $V_{j}$. This velocity is, practically, less than the ideal entering velocity (40$50 \mathrm{~km} / \mathrm{h}$ ), by $19 \mathrm{~km} / \mathrm{h}$, approximately [38]. Nevertheless, this method is appropriate for time calculation of straight routes only [6]. In this study, curved lanes are considered

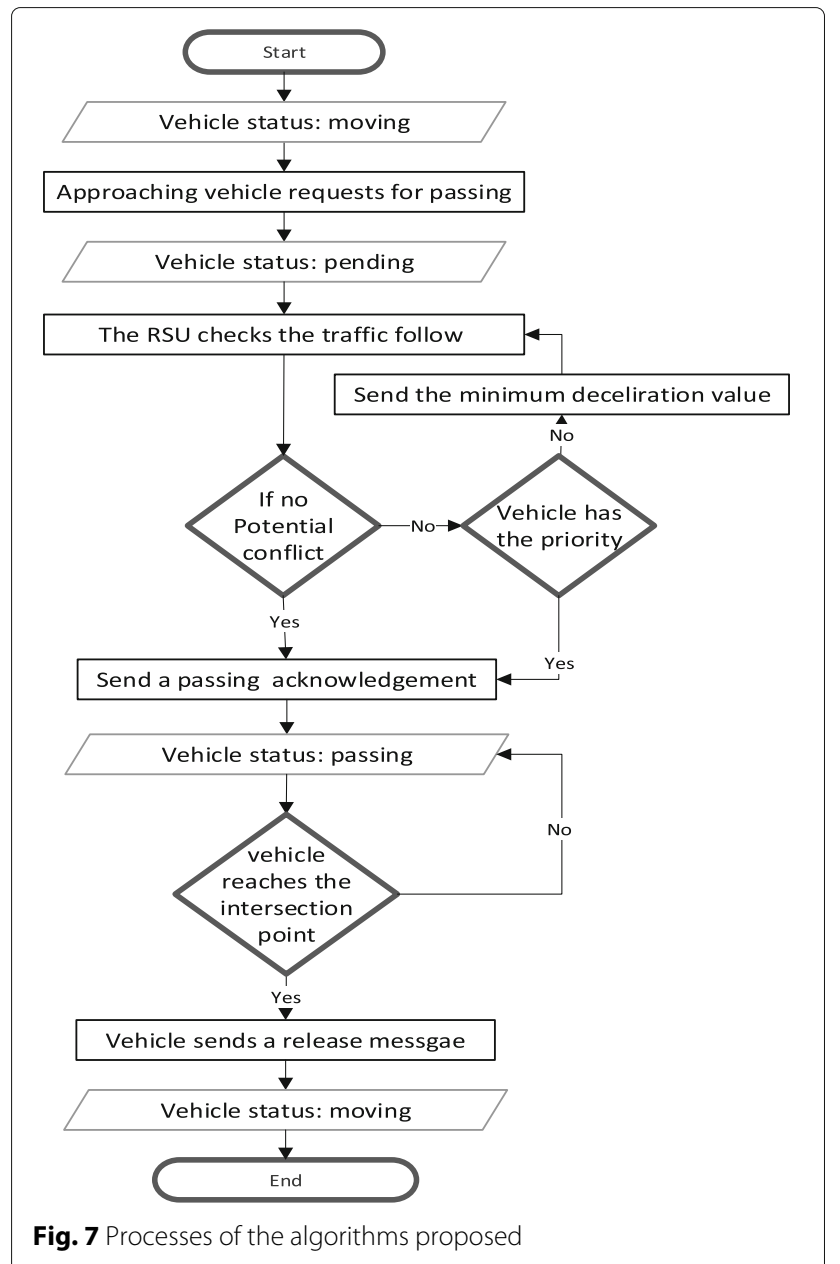

in computing the time elapsed, as shown in Fig. 8. Thus, time is expressed as:

$$
t_{i}=\frac{r_{i} \times \frac{\theta_{i}}{180} \times \Pi}{u}
$$

where $r_{i}$ denotes the turning radius of $V_{i}$ and $V_{j}$, and $\theta_{i}=2 \arcsin \left(\frac{c}{2 r_{i}}\right)$, for which $c$ refers to the line segment connecting both vehicles with the intersection line correlated.

On the other hand, if $V_{j}$ is not concurrent with $V_{i}$, then it has to pause while $V_{i}$ traverses the roundabout successfully. Hence, the pausing time of $V_{j}$ is expressed as:

$$
t_{j}=t_{i}-t_{\mathrm{vlc}}-\bar{t}_{j}
$$

where $t_{\mathrm{vlc}}$ denotes the delay of the VLC infrastructure and $\bar{t}_{j}$ denotes the time period $V_{i}$ takes to reach the intersection line to the time $V_{j}$ takes to reach the broadcast area. Consequently, $\bar{t}_{j}=0$ when $V_{j}$ already arrives at the stop line whereas $V_{i}$ is heading to the intersection line. Considering the ordinary roundabout traffic, one of the following scenarios is likely to occur when a vehicle reaches the roundabout broadcast area: 


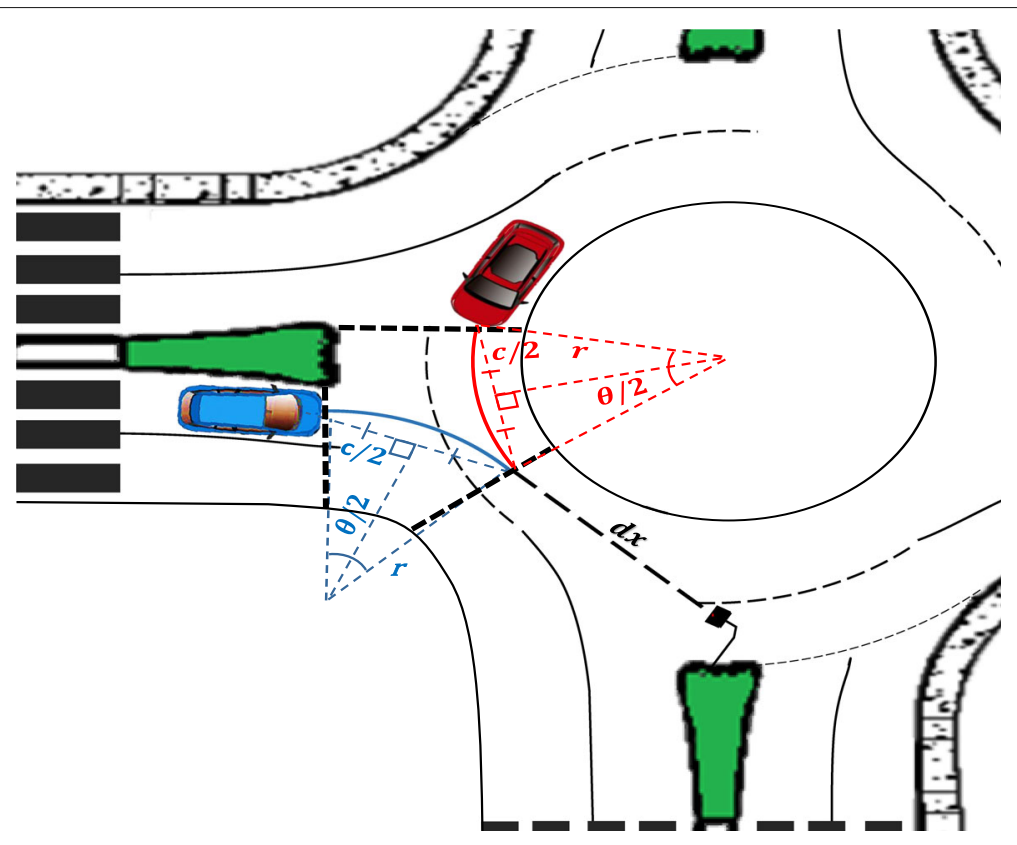

Fig. 8 Actual distances based on carved lanes

Case 1: The approaching vehicle reaches the roundabout firstly, meaning that no other vehicle is circulating at the roundabout, simultaneously.

Case 2: Vehicles are leaving the roundabout from the opposite path of the same leg.

Case 3: Another vehicle $V_{j}$ is circulating at the roundabout, simultaneously, in a path $P_{j}$ that is concurrent with the path $P_{i}$ of the approaching vehicle.

In the previous cases, the system will permit the approaching vehicle to move forward towards its destination, as the collision is unlikely to happen.

Case 4: Another vehicle $V_{j}$ is circulating at the roundabout, simultaneously, in a path $P_{j}$ that is asynchronous with the path $P_{i}$ of the approaching vehicle. Hence, $V_{j}$ is directed to decelerate, instantly, if the following state is true:

$$
\frac{d_{j}-d_{\mathrm{br}}}{v_{j}}+\frac{2 d_{\mathrm{br}}}{v_{j}+u}<t_{j}
$$

Since collision might occur, at the intersection line, among the two vehicles passing the asynchronous paths, by decelerating instantly, the collision is more likely to be waived. In the latter equation, $d_{j}$ denotes the area between $V_{j}$ and the correlated stop line and $v_{j}$ denotes the velocity of $V_{j}$. On the other hand, $V_{j}$ is not obliged to slow down when:

$$
\frac{d_{j}-d_{\mathrm{br}}}{v_{j}}+\frac{2 d_{\mathrm{br}}}{v_{j}+u}>=t_{j}
$$

where $V_{i}$ is more likely to pass the intersection line, smoothly, without colliding with the other vehicle.
To secure the safety, $V_{j}$ has to handle its driving velocity according to the ideal circulating speed $u$ at the end, by following the equation:

$$
v_{j}-a_{j} t_{j}<=u
$$

where $a_{j}$ denotes $V_{j}$ deceleration value being sent by the RSU within the acknowledgment message, as elaborated earlier in Algorithm 2

Considering the conditions mentioned above, $V_{j}$ deceleration distance must not transcend the stopping line presumed. The equation below expresses deceleration distance as:

$$
v_{j} t_{j}-\frac{1}{2} a_{j} t_{j}^{2}<=d_{j}
$$

\section{Results and discussion}

\subsection{Simulation parameters}

This section elaborates the results of the simulation performed to evaluate the performance of the system proposed. The simulation is performed via MATLAB depending on the theoretical analysis presented in the previous section. Hence, some parameters are considered for the sake of numerical calculation, as illustrated in Table 2.

During the simulation, the watt unit is converted to decibels $(\mathrm{dB})$ so that the analyses become more convenient. Moreover, the mobility of vehicles is considered when calculating the distance, where vehicles move towards the VLC emitter passing through the intersection point. Thus, the horizontal distance decreases from $71 \mathrm{~m}$ (the maximum value) to $30 \mathrm{~m}$, i.e., the intersection point 
Table 2 Simulation parameters of the warning system

\begin{tabular}{llll}
\hline Symbol & Description & Symbol & Description \\
\hline$d_{\text {total }}$ & $60 \mathrm{~m}$ & $d_{\mathrm{br}}$ & $10 \mathrm{~m}$ \\
$r$ & $35 \mathrm{~m}$ & $\mathrm{~N}$ & 4 \\
$h$ & $5.5 \mathrm{~m}$ & $\phi, \psi$ & $4.5^{\circ}$ \\
$T_{S}$ & 1 & $R$ & $0.4 \mathrm{~A} / \mathrm{W}$ \\
$I_{B}$ & $5 \times 10^{-3} \mathrm{~A}$ & $K$ & $1.38065 \times 10^{-23} \mathrm{~J} / \mathrm{K}$ \\
$T_{k}$ & $298 \mathrm{~K}\left(24.85^{\circ} \mathrm{C}\right)$ & $\eta$ & $112 \times 10^{-8} \mathrm{~F} / \mathrm{m}^{2}$ \\
$I_{2}$ & 0.562 & $I_{3}$ & 0.0868 \\
$B$ & $100 \mathrm{MHz}$ & $\mathrm{G}$ & 10 \\
$\Gamma$ & 1.5 & $g_{m}$ & $0.03 \mathrm{~S}$ \\
$t_{\mathrm{vlc}}$ & $0.5 \mathrm{~s}$ & $n$ & 1.46 \\
$q$ & $1.60218 \times 10^{-19} \mathrm{C}$ & $A$ & $1 \times 10^{-4} \mathrm{~m}^{2}$ \\
\hline$m$
\end{tabular}

$m$ meter, $A$ ampere, $W$ watt, $J$ joule, $K$ kelvin, $F$ farad, $M H z$ megahertz, $S$ Siemens, $C$ coulomb

location, by $1 \mathrm{~m}$. Considering the vehicle's hood, which is about $1 \mathrm{~m}$ in length, practically, the total workspace considered, i.e., less than $100 \mathrm{~m}$ in length, fits properly within the communication distance of the VLC technology [34].

\subsection{Simulation experiments}

To increase the efficiency and feasibility of the system proposed, the BER is plotted for different distances against various heights $(h)$ of the transmitter from the ground and various irradiance/incidence angles. Then, the best values are determined for the two parameters investigated as illustrated in Fig. 9. The simulation results in Fig. 9a exhibits that better BER value can be achieved by decreasing the height of the transmitter, considering the influence on the irradiation/incidence angle in each value. On the other hand, Fig. 9b exhibits that by choosing smaller irradiation/incidence angle, i.e., $4.5^{\circ}$, better BER can be achieved considering the optimal height at $5.5 \mathrm{~m}$.

Hence, the relationship between the distance variations and system's performance can be further studied considering the optimal values of the two parameters above mentioned, as shown in Fig. 10. First comes the relationship between distance and the received power. When considering the consistency of other parameters, the received power increases as the distance between the sender and receiver gets closer, due to the great light intensity at the receiver photodiode.

Second, the relationship between distance variations against SNR variations indicates that the minimum SNR value, which is $13.2986 \mathrm{~dB}$, is obtained at $71 \mathrm{~m}$, while the maximum SNR value $(28.0204 \mathrm{~dB})$ is obtained at $30 \mathrm{~m}$. This emphasizes that the quality of signal improves while the distance decreases toward the minimum value assumed. Finally, the figure presents the distance relationship over BER. It can be observed clearly that the BER value is directly proportional to the distance. Thus, the BER percentage approaches 0 at a minimum distance of $30 \mathrm{~m}$. On the other hand, it is the highest, i.e., $1.3280 \times$ $10^{-} 4$ at a distance of $71 \mathrm{~m}$.

Figure 11 shows the relationship between SNR and BER, which is inversely proportional, through exhibiting the SNR values along with its corresponding BER percentages, obtained from Eq. 12. For instance, when SNR = $13.2986 \mathrm{~dB}$, the corresponding $\mathrm{BER}=1.3280 \times 10^{-} 4$, which emphasizes that, at the same tested distance, the high SNR value results in a low BER percentage. According to our analytical results, establishing a LOS communication up to $71 \mathrm{~m}$, using the VLC technique, is feasible and it is adequate for a reliable communication link between the infrastructure and vehicles.

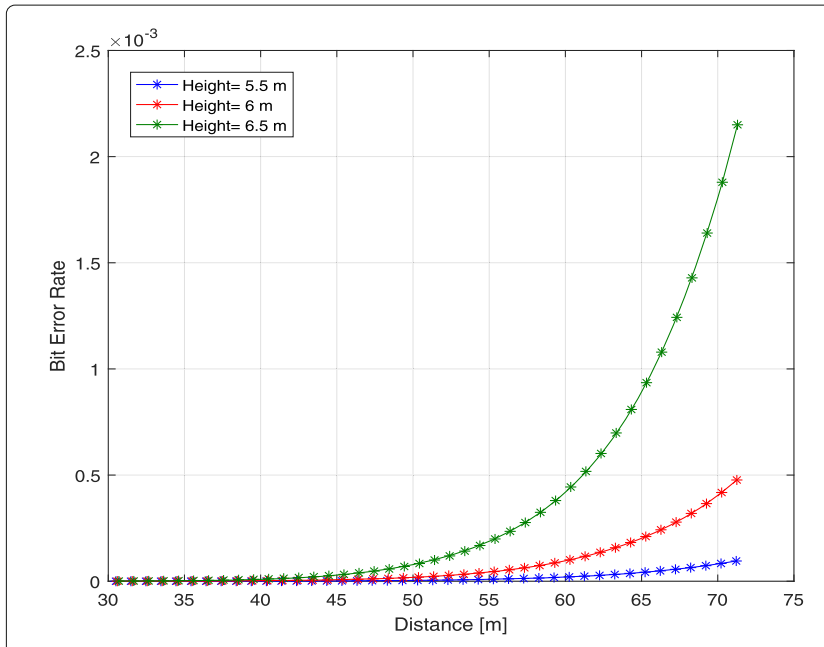

(a) Height effect on the distance with three distinctive values.

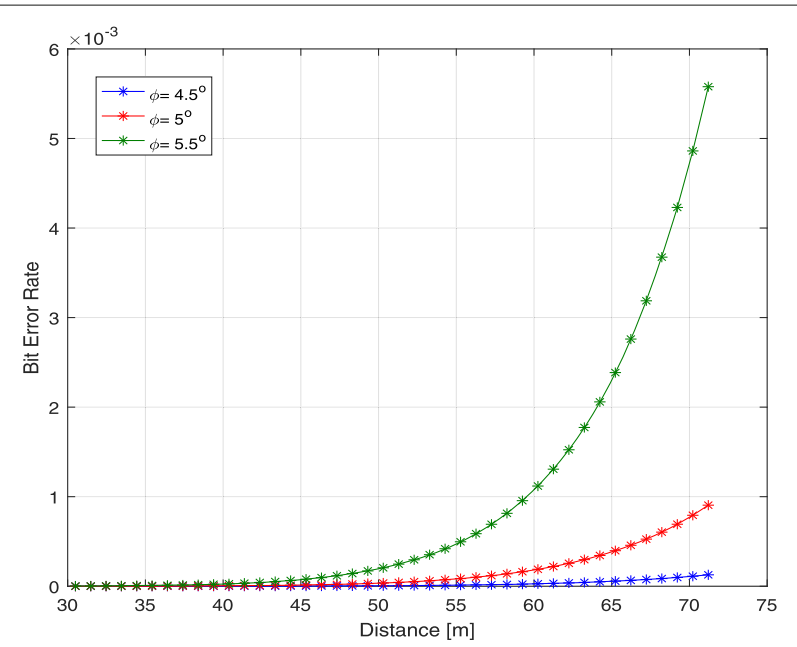

(b) Irradiation angle effect on the distance with three distinctive values.

Fig. 9 BER performance analysis of VLC system based for two effective parameters 


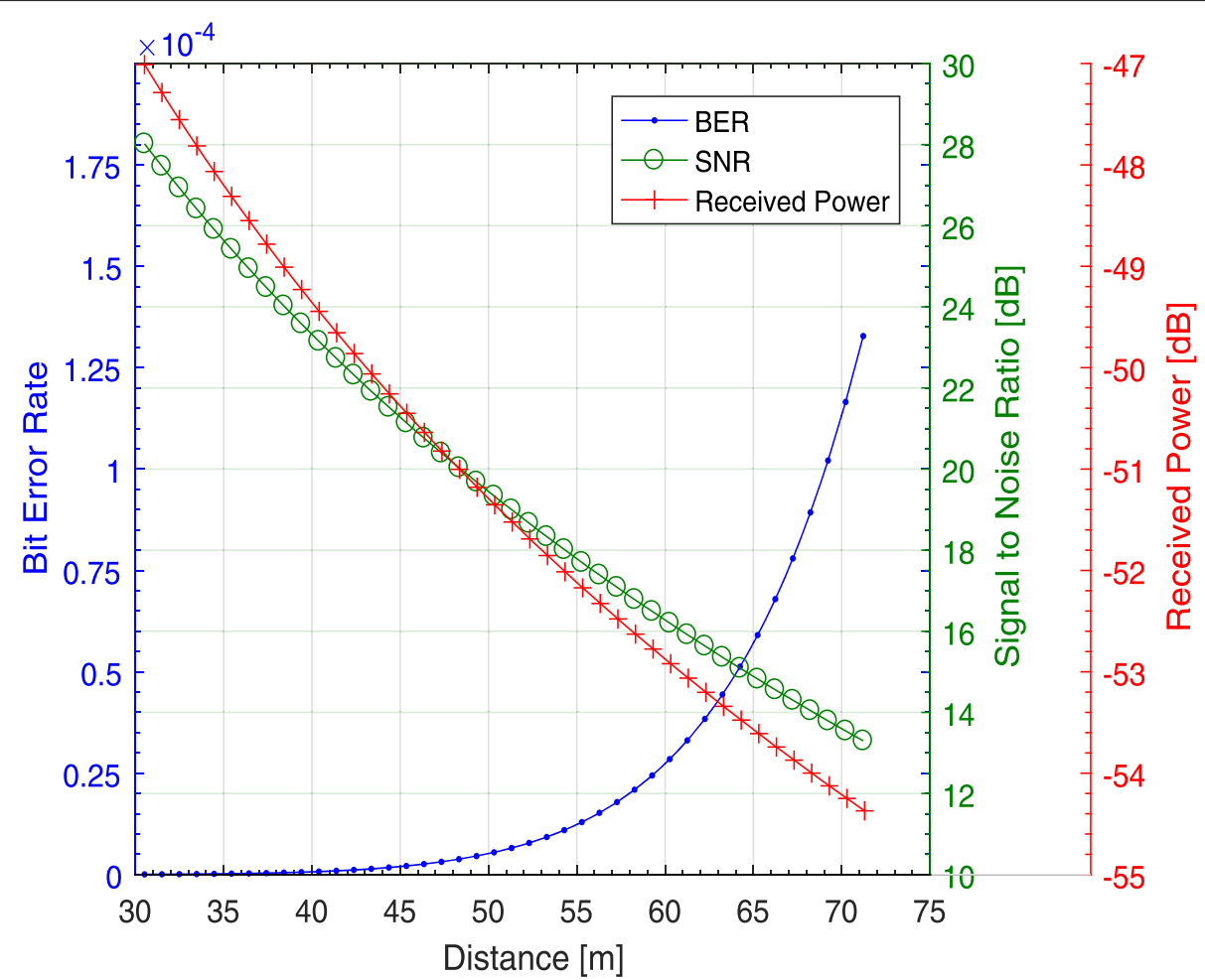

Fig. 10 BER, SNR, and received power at various distances using optimal parameter values

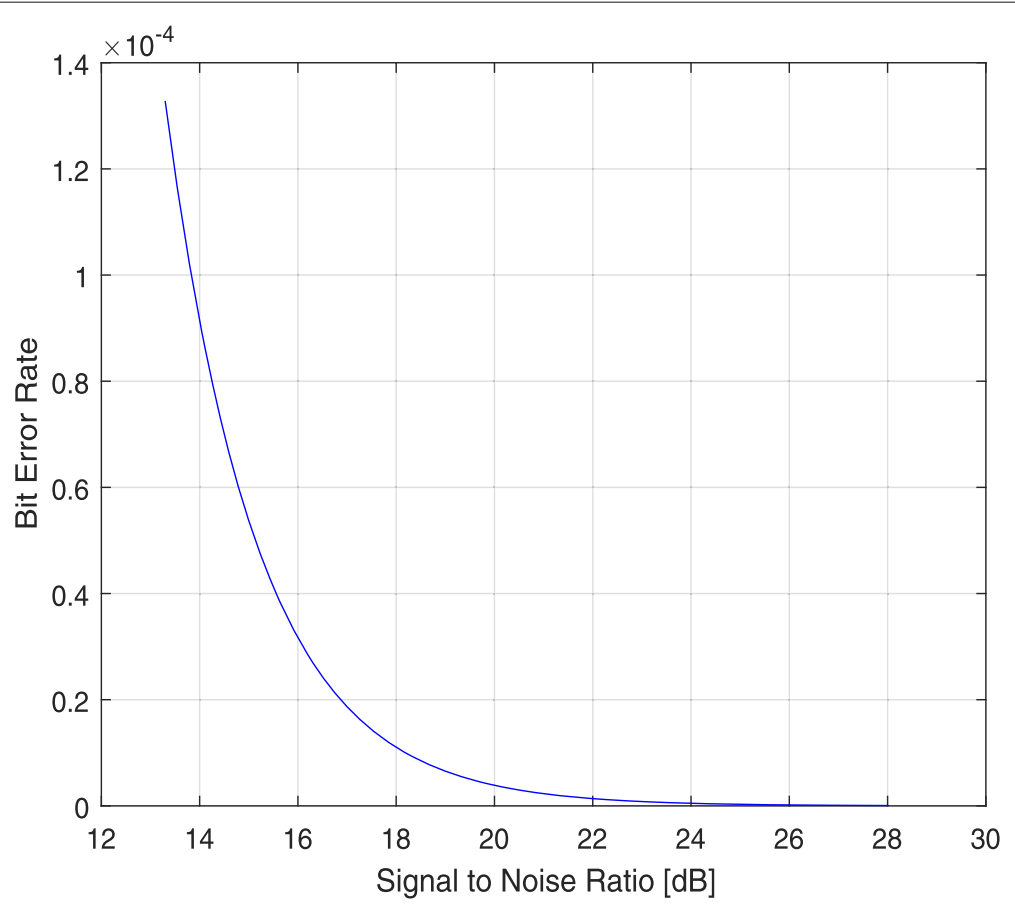

Fig. 11 Performance of the transmission as a function of BER and SNR 


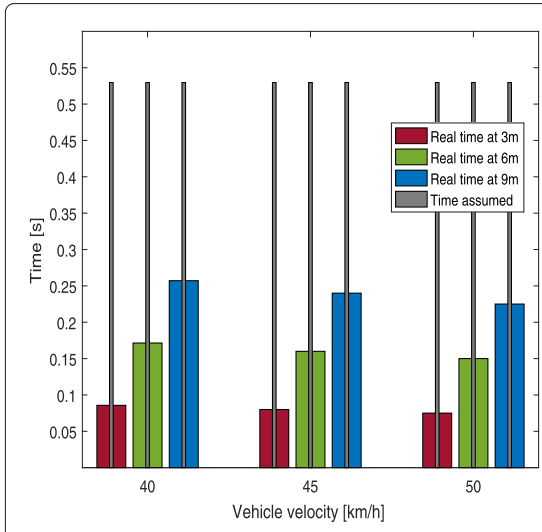

(a) $V_{j}$ is within the broadcast area.

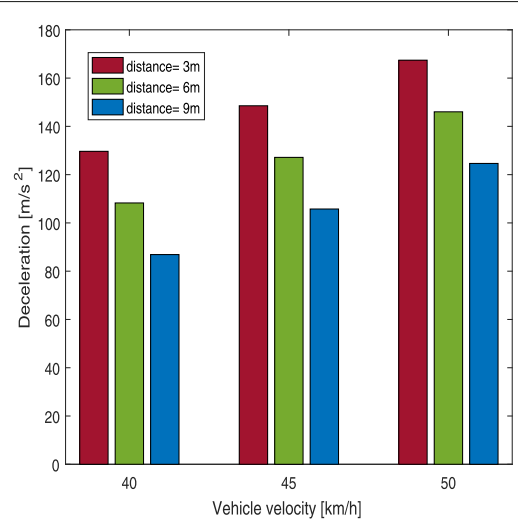

(b) minimum deceleration when $V_{j}$ is inside the broadcast area.

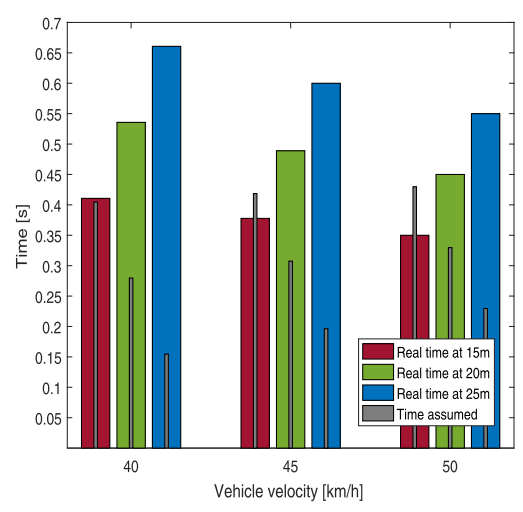

(c) $V j$ is outside the broadcast area.

Fig. $12 v_{j}$ patterns studied

To evaluate the system proposed efficiently, three driving velocities are assumed for vehicle $V_{j}$ (i.e., $40 \mathrm{~km} / \mathrm{h}$, $45 \mathrm{~km} / \mathrm{h}$, and $50 \mathrm{~km} / \mathrm{h}$ ), respectively. These velocities fall within the maximum entry velocities of multi-lane roundabouts. On the other hand, $V_{i}$ arrives at the stop line, firstly, after decreasing its driving speed to the ideal circulating speed $(30 \mathrm{~km} / \mathrm{h})$. Furthermore, two sets of patterns are identified, considering whether vehicle $V_{j}$ is located in or outside the roundabout broadcast area.

In the first pattern, the warning signal is being broadcasted by the RSU when vehicle $V_{j}$ is within the broadcast area, for which its present location is recorded. Consequently, three locations are assumed within the broadcast area ( $3 \mathrm{~m}, 6 \mathrm{~m}$, and $9 \mathrm{~m}$, consecutively).

Figure 12a confirmed that $V_{j}$ has to decelerate, instantly, in all positions tested within the broadcast area. Due to the fact that its practical time to arrive at the stop line is shorter than the time assumed since its position is close to the stop line. Accordingly, the information sent by the system is vital, as it includes the recommended minimum deceleration of $V_{j}$ to stop, precisely, at the stop line. Figure $12 \mathrm{~b}$ shows that the deceleration rate is directly proportional to $v_{j}$ and inversely proportional to $d_{j}$ as the deceleration value decreases with the decrease of the approaching velocity besides the increase of the stopping distance.

On the other hand, as assumed in the second pattern, the system sends the warning signal to $V_{j}$ when it is out of the broadcast area. In this paper, three positions are assumed out of the stopping line $(15 \mathrm{~m}, 20 \mathrm{~m}$, and $25 \mathrm{~m}$, consecutively) and $\bar{t}_{j}$ is being recorded, accordingly. Figure $12 \mathrm{c}$ exhibits that $V_{j}$ is strongly recommended to decelerate in $22 \%$ of the total cases only compared with the results in the first pattern, where $V_{j}$ real time is less than the time assumed, in most situations. For instance, $V_{j}$ is forced to decelerate if it is $5 \mathrm{~m}$ away from the broadcast area while driving at a velocity of $45 \mathrm{~km} / \mathrm{h}$.
Based on our results, potential collision within the roundabouts can be waived by using the proposed warning system since utilizing the VLC technique affects the system, positively, due to its high data rate and wide bandwidth. Furthermore, the VLC is less subject to wave interference, especially in high traffic scenarios, compared with conventional systems that utilize RF waves. Finally, no additional components are required, as these components are automatically supplied in today's vehicles.

\section{Conclusion}

VLC is an emerging technology used in outdoor environments related to ITS and road safety applications. This paper proposes a dynamic collision warning system that uses the VLC technique among autonomous vehicles as well as four RSUs deployed among the roundabout entrance legs. By this way, the system can control the traffic and grant pass permissions upon request to all vehicles approaching the roundabout, utilizing the principle of synchronization and priority according to the arrival time of vehicles. The experimentations demonstrate that the system proposed can wave potential collisions, efficiently.

As future work, the explicit priority of other particular vehicles will be considered (e.g., large trucks, emergency vehicles, and transit vehicles), in addition to applying our system on other types of intersections.

\section{Abbreviations}

VLC: Visible light communication; RSUs: Roadside units; V2l: Vehicle-to-infrastructure; ITS: Intelligent Transportation System; RF: Radio frequency; SNR: Signal-to-noise ratio; IM/DD: Intensity modulation with direct detection; V2V: Vehicle-to-vehicle; WSN: Wireless Sensor Networks; VMEl: Vehicle mutual exclusion for intersections problem; V2LC: Vehicular VLC network; UFSOOK: Undersampled Frequency Shift ON-OFF Keying modulation; BER: Bit error rate; AWGN: Additive white Gaussian noise; LOS: Line of sight; FOV :Field of view; OOK: On-Off Keying; OWC: Optical Wireless Communications; VIN: Vehicle Identification Number; GPS: Global Positioning System

Acknowledgements Not applicable. 


\section{Authors' contributions}

MF and SL proposed and deduced the main idea. MF worked on the overall writing of the paper. SL, XC and JZ worked on the manuscript review. All authors read and approved the final manuscript.

\section{Authors' information}

Maged Fakirah (maged_refat@daad-alumni.de) is a Ph.D. candidate in School of Information \& Communication Engineering, University of Electronic Science and Technology of China (UESTC), P.R. China. He received his Master degree in Computer Science from Jordan University of Science and Technology (JUST), Jordan. He worked as a part-time lecturer in Faculty of Computing and Information Technology, University of Science and Technology (UST), Yemen. His research interests include visible light communication, vehicular networks, Wireless Sensor Networks, Bioinformatics, and High-Performance Computing.

Supeng Leng (spleng@uestc.edu.cn) is a Full Professor and a Vice Dean in the School of Information \& Communication Engineering, University of Electronic Science and Technology of China (UESTC). He is also the leader of the research group of Ubiquitous Wireless Networks. He received his Ph.D. degree from Nanyang Technological University (NTU), Singapore. He has been working as a Research Fellow in the Network Technology Research Center, NTU. His research focuses on resource, spectrum, energy, routing and networking in Internet of Things, vehicular networks, broadband wireless access networks, smart grid, and the next-generation mobile networks. He published over 180 research papers in recent years. He serves as an organizing committee chair and TPC member for many international conferences, as well as a reviewer for over 10 international research journals.

Xiaosha Chen (pdcxs@outlook.com) received the B.Sc. degree in Information and Communication Engineering from University of Electronic Science and Technology of China, Chengdu, China, in 2016. He is currently working toward the Ph.D. degree in University of Electronic Science and Technology of China. His research interests include handling routing protocols, performance analysis, and congestion control in vehicular network.

Jihua Zhou (jhzhou@ict.ac.cn) is the assistant president and research fellow of Chongqing Jingmei Communication CO. LTD. He received his Ph.D. degree from the Institute of Computing Technology Chinese Academy of Sciences, China. His research interests include communication networks and mobile communications.

\section{Funding}

This work was partly supported by the National Key R\&D Program of China (No.2018YFE0117500), the Science and Technology Program of Sichuan Province, China (No.2019YFH0007), and the EU H2020 Project COSAFE (MSCA-RISE-2018-824019).

\section{Availability of data and materials}

Data sharing is not applicable to this article as no datasets were generated or analyzed during the current study.

\section{Competing interests}

The authors declare that they have no competing interests.

\section{Author details}

${ }^{1}$ School of Information \& Communication Engineering, University of Electronic Science and Technology of China (UESTC) Chengdu, China. ${ }^{2}$ Chongqing Jingmei Communication CO. LTD., Chongqing, China.

\section{Received: 1 November 2019 Accepted: 21 May 2020}

Published online: 16 June 2020

\section{References}

1. Q. Wang, S. Leng, H. Fu, Y. Zhang, An IEEE 802.11 p-based multichannel MAC scheme with channel coordination for vehicular ad hoc networks. IEEE Trans. Intell. Transp. Syst. 13(2), 449-458 (2011)

2. H. Zhou, X. Chen, S. He, C. Zhu, V. C. Leung, Freshness-aware seed selection for offloading cellular traffic through opportunistic mobile networks. IEEE Trans. Wirel. Commun. 19(4), 2658-2669 (2020)

3. H. Zhou, X. Chen, S. He, J. Chen, J. Wu, DRAIM: A novel delayconstraint and reverse auction-based incentive mechanism for WiFi offloading. IEEE J. Sel. Areas Commun. 38(4), 711-722 (2020)

4. Technology and Requirements for Self-Driving Cars. www.intel.com/ content/www/us/en/automotive/driving-safety-advanced-driver- assistance-systems-self-driving-technology-paper.html. Accessed Sept 2019

5. K.-H. N. Bui, D. Camacho, J. E. Jung, Real-time traffic flow management based on inter-object communication: a case study at intersection. Mob. Netw. Appl. 22(4), 613-624 (2017)

6. H. Cho, B.-w. Kim, Performance improvement of collision warning system on curved road based on intervehicle communication. Math. Probl. Eng. 2015(3), 1-7 (2015)

7. N. Lakouari, H. Ez-Zahraouy, A. Benyoussef, Traffic flow behavior at a single lane roundabout as compared to traffic circle. Phys. Lett. A. 378(43), 3169-3176 (2014)

8. L. A. Rodegerdts, Roundabouts: an informational guide. Transp. Res. Board. 672, 1-407 (2010). Washington, USA

9. M. Maurer, J. C. Gerdes, B. Lenz, H. Winner, et al, Autonomous Driving. Berlin, Germany: Springer Berlin Heidelberg. 10, 978-3 (2016)

10. C. Shao, S. Leng, Y. Zhang, A. Vinel, M. Jonsson, Performance analysis of connectivity probability and connectivity-aware MAC protocol design for platoon-based VANETs. IEEE Trans. Veh. Technol. 64(12), 5596-5609 (2015)

11. G. Qiao, S. Leng, K. Zhang, Y. He, Collaborative task offloading in vehicular edge multi-access networks. IEEE Commun. Mag. 56(8), 48-54 (2018)

12. X. Chen, S. Leng, Z. Tang, K. Xiong, G. Qiao, A millimeter wave based sensor data broadcasting scheme for vehicular communications. IEEE Access. 7, 149387-149397 (2019)

13. L. Cheng, W. Viriyasitavat, M. Boban, H.-M. Tsai, Comparison of radio frequency and visible light propagation channels for vehicular communications. IEEE Access. 6, 2634-2644 (2017)

14. M. Fakirah, S. Leng, Q. Wang, in 2019 IEEE 19th International Conference on Communication Technology (ICCT). Real-time traffic flow management based on visible light communication: a case study at roundabout (IEEE, Xi'an, 2019), pp. 690-694

15. A. Ndjiongue, H. C. Ferreira, An overview of outdoor visible light communications. Trans. Emerg. Technol. Technol. 29(7), 3448 (2018)

16. M. Lèbre, F. Le Mouël, E. Ménard, in 2016 83rd Vehicular Technology Conference (VTC Spring). Resilient, decentralized V2V online stop-free strategy in a complex roundabout (IEEE, Nanjing, 2016), pp. 1-5

17. R. Azimi, G. Bhatia, R. Rajkumar, P. Mudalige, V2v-intersection management at roundabouts. SAE Int. J. Passenger Cars-Mech. Syst. 6(2013-01-0722), 681-690 (2013)

18. G. Tan, Y. Wang, Y. Wang, H. Ge, in 2011 Third International Conference on Intelligent Human-Machine Systems and Cybernetics. A new traffic-signal control for large multi-branch roundabouts (IEEE, Zhejiang, 2011), pp. 7-11

19. W. Wu, J. Zhang, A. Luo, J. Cao, Distributed mutual exclusion algorithms for intersection traffic control. IEEE Trans. Parallel Distrib. Syst. 26(1), 65-74 (2014)

20. M. Elhadef, in 2015 IEEE International Conference on Computer and Information Technology; Ubiquitous Computing and Communications; Dependable, Autonomic and Secure Computing; Pervasive Intelligence and Computing. An adaptable inVANETs-based intersection traffic control algorithm (IEEE, Liverpool, 2015), pp. 2387-2392

21. Y. Wang, K. Wang, H. Huang, T. Miyazaki, S. Guo, Traffic and computation co-offloading with reinforcement learning in fog computing for industrial applications. IEEE Trans. Ind. Inform. 15(2), 976-986 (2018)

22. X. He, K. Wang, H. Huang, T. Miyazaki, Y. Wang, S. Guo, Green resource allocation based on deep reinforcement learning in content-centric loT. IEEE Trans. Emerg. Top Comput. (2018)

23. K. Wang, Q. Zhou, S. Guo, J. Luo, Cluster frameworks for efficient scheduling and resource allocation in data center networks: a survey. IEEE Commun. Surv. Tutorials. 20(4), 3560-3580 (2018)

24. B. M. Masini, A. Bazzi, A. Zanella, Vehicular visible light networks for urban mobile crowd sensing. Sensors (Switzerland). 18(4), 1177 (2018). https:// doi.org/10.3390/s 18041177

25. C. B. Liu, B. Sadeghi, E. W. Knightly, Enabling Vehicular Visible Light Communication (V2LC) Networks (June), (Las Vegas, 2011), pp. 41-50. https://doi.org/10.1145/2030698.2030705

26. P. Ji, H. M. Tsai, C. Wang, F. Liu, Vehicular visible light communications with LED taillight and rolling shutter camera. IEEE Veh. Technol. Conf. 2015, 1-6 (2014). https://doi.org/10.1109/NTCSpring.2014.7023142

27. M. S. Ab-Rahman, N. I. Shuhaimi, L. A. Azizan, M. R. Hassan, Analytical study of signal-to-noise ratio for visible light communication by using single source. Jo Comput. Sci. 8(1), 141 (2012) 
28. Z. Ghassemlooy, W. Popoola, S. Rajbhandari, Optical Wireless Communications: System and Channel Modelling with Matlab ${ }^{\circledR}$. (CRC press, boca Raton, 2012)

29. C. Chen, W.-D. Zhong, H. Yang, S. Zhang, P. Du, Reduction of SINR fluctuation in indoor multi-cell VLC systems using optimized angle diversity receiver. J. Light. Technol. 36(17), 3603-3610 (2018)

30. Y. Qiu, H.-H. Chen, W.-X. Meng, Channel modeling for visible light communications-a survey. Wirel. Commun. Mob. Comput. 16(14), 2016-2034 (2016)

31. Y. Chen, C. W. Sung, S.-W. Ho, W. S. Wong, BER analysis and power control for interfering visible light communication systems. Optik- Int. J. Light Electron. Opt. 151, 98-109 (2017)

32. W. Stallings, Data and Computer Communications (8th Edition), Prentice-Hall, Inc., USA (2006)

33. S.-H. Lin, J.-Y. Wang, X. Bao, Y. Li, Outage and bit error rate analysis for vehicular visible light communications. Opt. Eng. 56(8), 086114 (2017)

34. S. Rajagopal, R. D. Roberts, S.-K. Lim, IEEE 802.15. 7 visible light communication: modulation schemes and dimming support. IEEE Commun. Mag. 50(3), 72-82 (2012)

35. S. Arnon, Visible Light Communication. (Cambridge University Press, Cambridge, 2015)

36. Y. Cao, T. Jiang, O. Kaiwartya, H. Sun, H. Zhou, R. Wang, Toward pre-empted EV charging recommendation through $\mathrm{V} 2 \mathrm{~V}$-based reservation system. IEEE Trans. Syst. Man, Cybern. Syst. (2019)

37. A. M. Cailean, M. Dimian, Current challenges for visible light communications usage in vehicle applications: a survey. IEEE Comput. Surv. Tutorials. 19(4), 2681-2703 (2017). https://doi.org/10.1109/COMST. 2017.2706940

38. WSDOT, Design Manual M 22-01.18, Chapter 1320 - Roundabouts, December, Olympia, Washington, USA, 1-28 (2019)

\section{Publisher's Note}

Springer Nature remains neutral with regard to jurisdictional claims in published maps and institutional affiliations.

\section{Submit your manuscript to a SpringerOpen ${ }^{\circ}$ journal and benefit from:}

- Convenient online submission

- Rigorous peer review

- Open access: articles freely available online

- High visibility within the field

- Retaining the copyright to your article

Submit your next manuscript at $>$ springeropen.com 